\title{
Evaluating Intelligent Search Agents in a Controlled Environment Using Complex Queries: An Empirical Study
}

\author{
Nurul I Sarkar ${ }^{1}$, Xiumin Wang ${ }^{2}$, Liang Shan ${ }^{2}$, Bo Hong ${ }^{2}$ and ${\mathrm{Jun} \mathrm{Li}^{2}}^{2}$ \\ ${ }^{1}$ Department of Information Technology and Software Engineering, Auckland \\ University of Technology, New Zealand \\ ${ }^{2}$ Department of Information Engineering, China Jiliang University, \\ Hangzhou, China, 310018 \\ wxm6341@163.com(Corresponding author)
}

\begin{abstract}
There is a growing interest in using intelligent search agents (ISAs) in e-commerce and online businesses worldwide in recent years. This interest results from the availability of various sophisticated and powerful intelligent agents that can automate the process of searching through and evaluating reams of information on the Web. While efforts have been made recently to develop various powerful ISAs and multi-agent systems, very little is understood and known about evaluation of such agents. In this paper we describe a simple experimental setup ('system') that can be used to evaluate ISAs without using any complex algorithm and mathematical analysis. The idea is to evaluate search agents based on a performance metric in a controlled environment using complex search queries. For an efficient evaluation of ISAs, we introduce a new metric called 'search speed' which is a ratio of the number of results returned per second per query to the round trip time. This paper provides an in-depth performance comparison of four selected ISAs (Copernic, FirstStop Websearch, iMeta, and WebFerret). These search agents were selected based on their availability, popularity, and interesting characteristics and features. The analysis and empirical results reported in this paper provide some insights into the evaluation of ISAs which may help researchers to evaluate similar search agents.
\end{abstract}

Keywords: Intelligent search agents, performance evaluation, search speed

\section{Introduction}

The competitive business environment and the growing complexity of work and personal lives require many tasks to be performed (often simultaneously) more efficiently and promptly. To support these imperatives, new sophisticated and powerful intelligent software tools have been developed. One such emerging software tool is the notion of an ISA [1-3].

ISAs and multi-agents are becoming increasingly popular in applications such as process management, manufacturing and distributed systems, routing in mobile ad hoc networks, database administration, financial application, e-commerce, and online businesses [4-8]. This popularity is due to the availability of various sophisticated and powerful intelligent agents that can automate the process of information searching and evaluating on the Web. For example, an ISA can be used to search on the Web to find a car matching a list of criteria, as tracking down the best price for purchasing grey Toyota Corolla 2012.

A detailed discussion of intelligent agents, in general, can be found in agent technology literature [9-14]. Rimmel et al., [15] categorize intelligent agents based on their operating environment, such as desktop agents, network agents, web search agents, filter agents,

Received (October 19, 2017), Review Result (December 5, 2017), Accepted (December 10, 2017) 
memory agents, and service agents. Nwana [13] classifies intelligent agents on the basis of their primary attributes, such as mobility (mobile agents) and cooperation (collaborative agents). Mobile agents can travel through the Internet and gather information by interacting with information databases and other agents. Collaborative agents accentuate on autonomy and cooperation with other agents to fulfil its task. Wan et al., [16] propose a conceptual method for classifying product comparison agents based on the ecological food chain.

Although many sophisticated intelligent agents and multi-agents systems have been developed in recent years, an empirical evaluation of such agents is still an unexplored area in the field of agent technology [17-18]. Indeed, an accurate evaluation of ISAs can be a complex task because of various factors (both controlled and uncontrolled) involved in the process. This paper describes an experimental setup for evaluating ISAs in a controlled environment using complex search queries. The main contributions are:

a) A thorough review of literature on ISAs is presented.

b) A simple but effective experiment setup for evaluating ISAs is presented. We introduce a new metric called 'search speed' for efficient evaluation of ISAs.

c) An in-depth comparison of Copernic, FirstStop Websearch, iMeta, and WebFerret using complex search queries to verify the effectiveness of the proposed system is presented.

\section{Research methodology and question}

This research is exploratory in the sense that there was very limited prior research in the area of performance evaluation of ISAs to guide this research endeavor. Therefore, we adopted an empirical methodology for evaluating ISAs [19-20]. The following research question was proposed.

Given the two or more similar agents, how can we tell which one is better and/or faster without going through complex mathematical analysis? In other words, how can we select the best search agent from a pool of search agents?

To address the above question, we quantitatively analyze the performance of four selected ISAs, Copernic Agent v6.2, FirstStop Websearch 5, iMeta Search 5, and WebFerret 6.0.

\section{Internet Search Agents: a Review of Literature}

A number of recent studies demonstrate the usefulness and viability of using agentbased technologies in various applications; for example, in manufacturing automation [6][21-22], managing relational database [23] network payment security [24], e-business [8][25], data mining and information retrieval [26], company knowledge management [27-28], fault diagnose system [29], warehouse control [30], 3G mobile system, education [31-32], agent-based intelligent manufacturing shop floor [33], radar systems [34], and web content filtering and information gathering [35-37].

Mawlood-Yunis et al., [38] experimented with two mobile agents in evaluating the behaviour of the agent's platform in distributed search environment. An interesting finding of this research is that in small networks multi-agent systems do not provide performance advantages because of the high overheads.

Camacho et al., [39] evaluated various agent platforms (e.g., JADE, ZEUS, and SKEKETONAGENT) based on the performance metrics, such as the number of agents, requested documents, request time, and the number of articles retrieved. Oi and Sun [40] identified operators that may increase the performance of a multi-agent system.

Lau and Zhang [41] investigated the agents' cooperation in a multi-agent system. It has been shown that the partial cooperation between two agents is better than the fully 
cooperated agents. Dora [19] highlights the importance of using multiple criteria for the evaluation of intelligent agents. Mao et al., [42] point out that the coordination amongst the agents in a multi-agent system effects the system performance.

\section{Description of Experiment Setup}

Figure 1 shows an overview of ISAs evaluation system. It allows users to conduct various experiments for the performance evaluation and comparison of ISAs based on a selected performance metric. To obtain an accurate evaluation results, it is important to use an appropriate performance metric. The system generates evaluation results that can be analysed later for the selection of the best agent for a particular scenario/application.

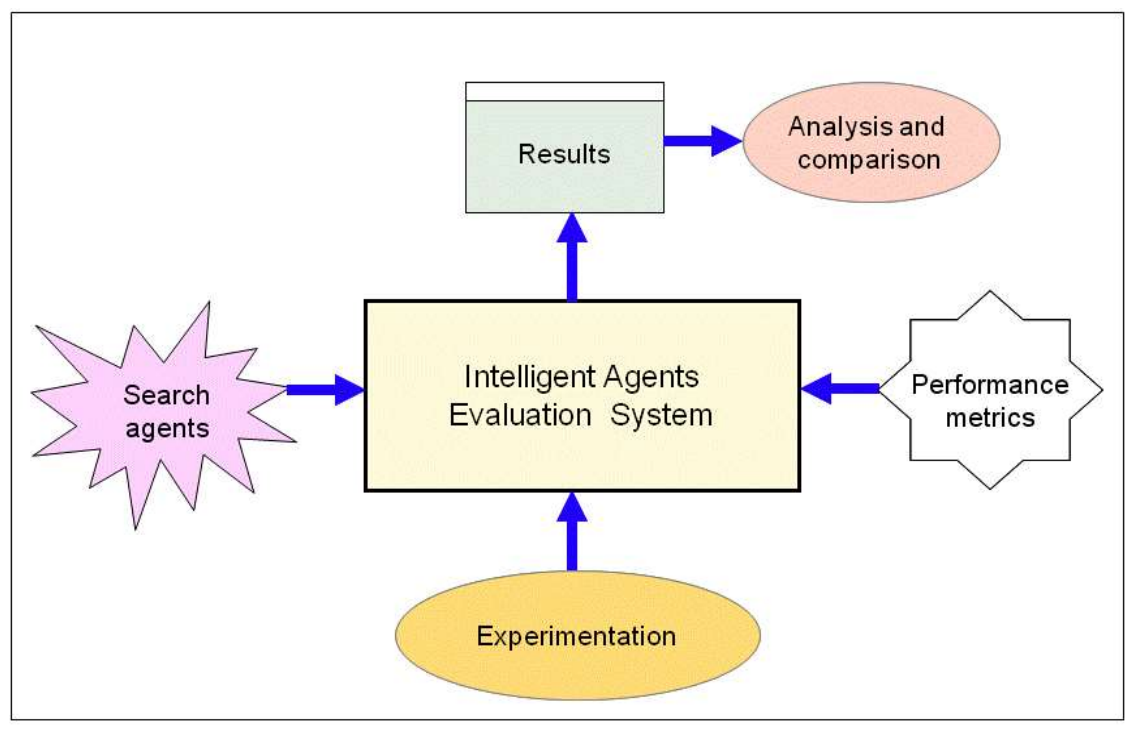

Figure 1. The Proposed Agent Evaluation System

\section{Performance Metrics}

While various metrics for the performance evaluation of intelligent agents are reported in the agent technology and software engineering literature, we briefly describe the following commonly used performance metrics that we have used in the experiments.

a) Throughput: This metric (measured in bytes/sec) is commonly used for performance measurement of computer and data communication networks. In the evaluation of ISAs, the network throughput was measured for all iterations performed ensuring that the network throughput was not affecting the performance measures significantly.

b) Round trip time (RTT): The RTT (measured in seconds) is the time measured from the moment a query is placed on a source machine until the query arrives. The RTT can be affected by a search query size.

c) Results returned: This metric gives us the total number of results returned by an ISA after completing a search. The 'results returned' and RTT are used to define a new metric called 'search speed'. While the 'number of results returned' and 'RTT' can be useful for the performance measurements of ISAs, it is often difficult to make a final conclusion about the capability of an agent just by considering these two metrics independently. Therefore, we define 'search speed' (denoted by $\mathrm{S}_{\text {speed}}$ ) by combing results returned and RTT as follows:

$$
S_{\text {speed }}=\frac{\text { total_number_of_serach_results_returned }}{R T T(\sec \text { onds })}
$$


The $\mathrm{S}_{\text {speed }}$ tells us about the capacity of an agent (i.e., how many results returned by an agent per second per query). An agent is said to be more powerful if it has a higher $S_{\text {speed }}$. This metric is used in this study for an efficient performance evaluation of ISAs.

d) Memory consumed: To measure the amount of computer memory consumed (measured in $\mathrm{KB}$ ) by an ISA, the MS Windows ${ }^{\circledR}$ default task manager utility was used. The memory usage is measured dynamically when an application runs on the system.

e) Accuracy: In information retrieval, accuracy is measured in terms of recall and precision, where recall is relevance of the results obtained and precision is the cleanliness of the results [43].

\section{Experiment Details}

Figure 2 shows the experimental set-up for the performance evaluation of ISAs. The experimental setup consists of a desktop PC (Intel Pentium 4, $2.8 \mathrm{GHz} ; 512 \mathrm{MB}$ RAM; 60 GB hard disk; Windows XP Professional) and an ADSL modem (upload speed: 128 Kbps; download speed: $2 \mathrm{Mbps}$ ) linked to the Internet. To measure the RTT and throughput, an open source tool called Wireshark was used to capture packets from the live network.

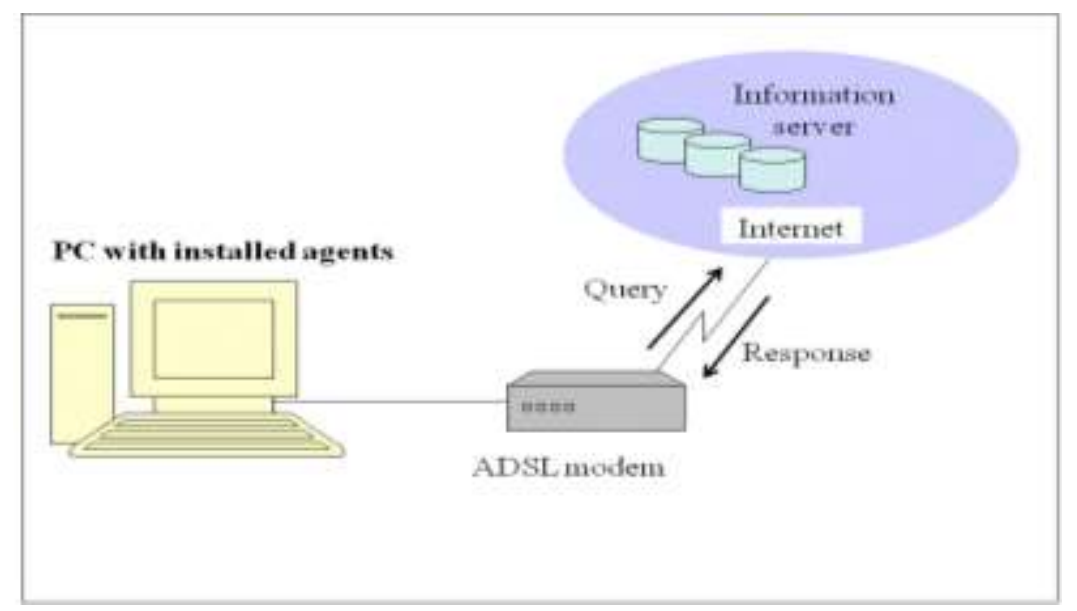

\section{Figure 2. An Experimental Setup for Evaluating Search Agents}

The selection of queries was very crucial in our experiments. According to Jansen [44] most of the web queries are only of two terms, where a term is a string of unbroken characters, or a series of characters. A term can be a word, number, symbols, or even a URL. A query also contains one or more search terms and logical operators. In the experimentation we have used more complex queries with respect to higher search terms. Table 1 lists queries with 4, 5, 6, and 7 terms.

Table 1. Queries used in the Experiments

\begin{tabular}{cl}
\hline Number of terms & Query \\
\hline 4 & How to make pudding \\
5 & Who said: "now or never" \\
6 & Information on colleges located in Germany \\
7 & Information on effects of caffeine on heart \\
\hline
\end{tabular}

The selection of ISAs was a multifaceted and complex task as we had very limited ISAs available for download at no costs to conduct experiments under a control 
environment. Most of the agents available on the Web are the ones in which users can submit a query online and the agent provides the results. Table 2 describes the four ISAs that we have evaluated. To obtain more accurate and unbiased results, each query was submitted to each of the four selected ISAs in turn for 10 times and all the measurements were recorded. We repeated each experiment 10 times because it is a realistic figure that provided satisfied outcome. We collected data at three different times of the day: 9am, $4 \mathrm{pm}$, and $9 \mathrm{pm}$. In summary, we took the mean (arithmetic average) of 30 observations per query per ISA.

Table 2. Four selected ISAs and their Description

\begin{tabular}{l|l}
\hline Search agents & \multicolumn{1}{c}{ Description } \\
\hline CopernicAgent v6.2 & $\begin{array}{l}\text { This agent has the capability of searching multiple sources. It } \\
\text { combines the results and removes duplicates for each search. }\end{array}$ \\
\hline $\begin{array}{l}\text { FirstStop } \\
\text { Websearch 5 }\end{array}$ & $\begin{array}{l}\text { This agent has the capability of searching through multiple } \\
\text { engines concurrently. It provides a comprehensive search results. }\end{array}$ \\
\hline iMeta Search 5 & $\begin{array}{l}\text { It has the capability of searching through multiple sources, index } \\
\text { and sorting the results. }\end{array}$ \\
\hline WebFerret 6.0. & $\begin{array}{l}\text { This agent can search through multiple sources concurrently. It } \\
\text { provides search results in detail, can be used as a shopping agent. }\end{array}$ \\
\hline
\end{tabular}

\section{Results and Discussion}

We consider the following five performance metrics for the evaluation of Copernic, FirstStop, iMeta, and WebFerret: (1) memory consumed; (2) round trip time (RTT); (3) throughput; (4) results returned; and (5) search speed. In this section we present numerical results obtained from empirical study. The mean (average) memory consumed, RTT, throughput, number of results returned, and search speed of the five selected ISAs are summarized in Table 3.

We now compare the performance of Copernic, FirstStop, iMeta, and WebFerret against each of the performance metrics in turn. In Figure 3, we plot ISAs versus mean memory consumed. The mean memory consumed by an individual ISA was measured by executing the search tasks for all three queries as listed in Table 1 . Recall that each query was submitted 10 times to each of the ISA at three different times. Therefore, the mean memory consumed (by an ISA) is the arithmetic average of 90 observations (Figure 3). Of the four ISAs, WebFerret consumes the lowest memory $(<4000 \mathrm{kB})$ and iMeta consumes highest $(>9000 \mathrm{kB})$.

The RTT performance of Copernic, FirstStop Websearch, iMeta, and WebFerret is shown in Figure 4. We observed that iMeta performs better than the other three ISAs.

Table 3. A Summary of ISAs Evaluation Results

\begin{tabular}{|c|c|c|c|c|c|}
\hline \multicolumn{7}{|c|}{$\begin{array}{c}\text { Experiment } \\
\text { conducted }\end{array}$} & $\begin{array}{c}\text { Memory } \\
\text { consumed (KB) }\end{array}$ & $\begin{array}{c}\text { RTT } \\
\text { (sec) }\end{array}$ & $\begin{array}{c}\text { Throughput } \\
\text { (bytes/sec) }\end{array}$ & $\begin{array}{c}\text { \# of results } \\
\text { returned (NRR) }\end{array}$ & $\begin{array}{c}\text { Search speed } \\
\text { (NRR/sec/query) }\end{array}$ \\
\hline Morning & 8465.7 & 14 & 601960 & 65.5 & 5.3 \\
\hline Afternoon & 9296.2 & 12.6 & 598535 & 65.2 & 6.1 \\
\hline Night & 8353.3 & 12.8 & 600573 & 64.7 & 5.3 \\
\hline Mean & 8705.1 & 13.2 & 600356 & 65.1 & 5.6 \\
\hline \multicolumn{7}{|c|}{$\begin{array}{c}\text { FirstStop } \\
\text { Experiment } \\
\text { conducted }\end{array}$} & $\begin{array}{c}\text { Memory } \\
\text { consumed (KB) }\end{array}$ & $\begin{array}{c}\text { RTT } \\
\text { (sec) }\end{array}$ & $\begin{array}{c}\text { Throughput } \\
\text { (bytes/sec) }\end{array}$ & $\begin{array}{c}\text { \# of results } \\
\text { returned (NRR) }\end{array}$ & $\begin{array}{c}\text { Search speed } \\
\text { (NRR/sec/query) }\end{array}$ \\
\hline
\end{tabular}




\begin{tabular}{|c|c|c|c|c|c|}
\hline Morning & 6124 & 7.2 & 421432.2 & 20 & 3.6 \\
\hline Afternoon & 6504.6 & 7.6 & 426470.2 & 21 & 3.4 \\
\hline Night & 6035 & 6.8 & 415491.6 & 20 & 3.8 \\
\hline Mean & 6221.2 & 7.2 & 421131.3 & 20.3 & 3.6 \\
\hline \multicolumn{5}{|c|}{ WebFerret } \\
\hline $\begin{array}{c}\text { Experiment } \\
\text { conducted }\end{array}$ & $\begin{array}{c}\text { Memory } \\
\text { consumed (KB) }\end{array}$ & $\begin{array}{c}\text { RTT } \\
\text { (sec) }\end{array}$ & $\begin{array}{c}\text { Throughput } \\
\text { (bytes/sec) }\end{array}$ & $\begin{array}{c}\text { \# of results } \\
\text { returned (NRR) }\end{array}$ & $\begin{array}{c}\text { Search speed } \\
\text { (NRR/sec/query) }\end{array}$ \\
\hline Morning & 3830.7 & 16.6 & 816474.9 & 70.3 & 6.5 \\
\hline Afternoon & 3788.4 & 19.2 & 799664.2 & 69.3 & 4.8 \\
\hline Night & 3769.9 & 12.7 & 744608.4 & 51.0 & 6.1 \\
\hline Mean & 3796.4 & 16.2 & 786915.8 & 63.5 & 5.8 \\
\hline \multicolumn{7}{|c|}{} & iMeta & \multicolumn{1}{|c|}{} \\
\hline $\begin{array}{c}\text { Experiment } \\
\text { conducted }\end{array}$ & $\begin{array}{c}\text { Memory } \\
\text { consumed (KB) }\end{array}$ & $\begin{array}{c}\text { RTT } \\
\text { (sec) }\end{array}$ & $\begin{array}{c}\text { Throughput } \\
\text { (bytes/sec) }\end{array}$ & $\begin{array}{c}\text { \# of results } \\
\text { returned (NRR) }\end{array}$ & $\begin{array}{c}\text { Search speed } \\
\text { (NRR/sec/query) }\end{array}$ \\
\hline Morning & 9191 & 4.9 & 75683.2 & 20 & 7.8 \\
\hline Afternoon & 10118.9 & 5.6 & 77089.5 & 20 & 8.5 \\
\hline Night & 9144.5 & 5.0 & 75797.8 & 20 & 8.8 \\
\hline Mean & 9484.8 & 5.1 & 76190.2 & 20 & 8.4 \\
\hline
\end{tabular}

In Figure 5, we plot ISAs versus mean network throughput to observe the status of the network throughput. We observed that the network throughput varies ranging from 121,422 to 136,191 bytes/second. As shown in Figure 5, the network had the highest throughput during WebFerret's performance measurements. The network throughput was measured for all iterations performed in the experimentation. However, every effort has been made to minimize the impact of network throughput on the performance of ISAs, but it was not always possible to keep the network throughput constant at all times during experimentation. Therefore, we repeat the same experiment at different times of the day to obtain the mean network throughput.

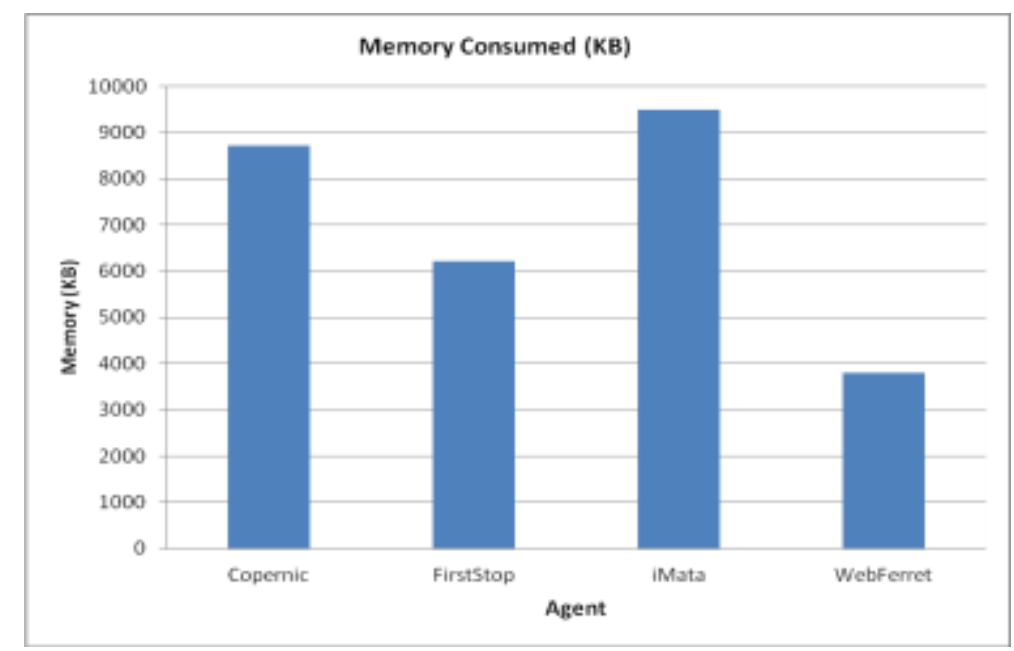

Figure 3. Comparison Memory Consumed by Copernic, FirstStop, iMeta, and WebFerret 


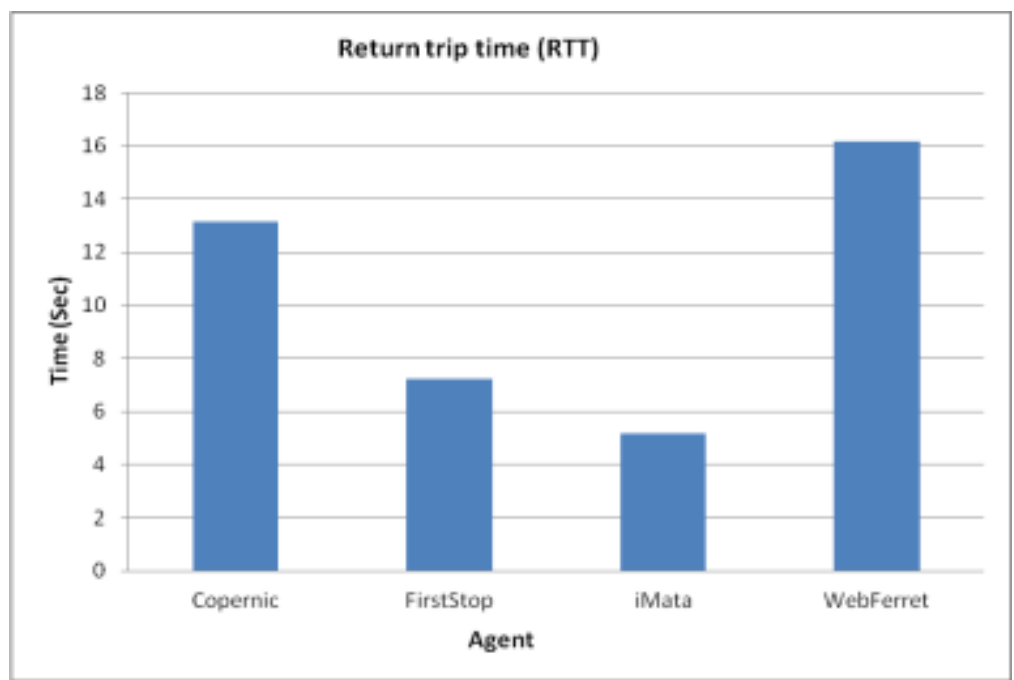

Figure 4. Comparison of RTT for Copernic, FirstStop, iMeta, and WebFerret

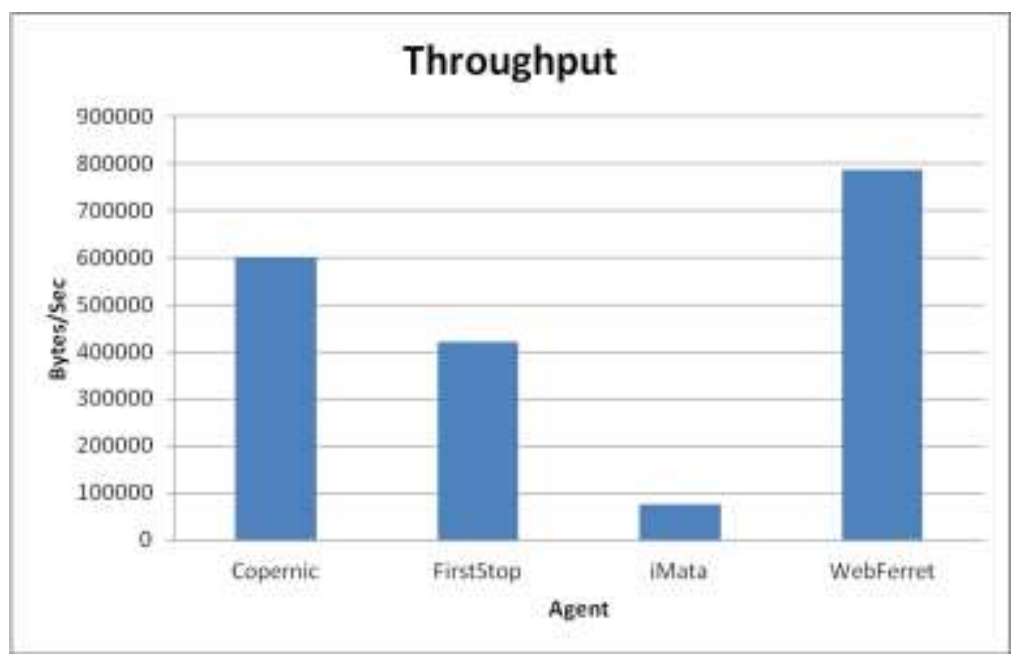

Figure 5. Throughput Comparison of Copernic, FirstStop, iMeta, and WebFerret

The mean number of results returned after completing the search by Copernic, FirstStop Websearch, iMeta, and WebFerret is shown in Figure 6. We found that Copernic performs better with respect to the number of results returned per second per query than the FirstStop, iMeta, and WebFerret. In Figure 7, we plot ISAs versus mean search speed. Recall that the "search speed" is the ration of "number of results returned per second per query" and RTT. The search speed allows us to determine the capacity of an ISA. Of the four ISAs, iMeta has the highest and FirstStop has the lowest search speed. The main conclusion that can be drawn from Figures 3 to 7 is that iMeta is the most powerful search agent among the four ISAs evaluated, despite of unfavorable network throughput. 


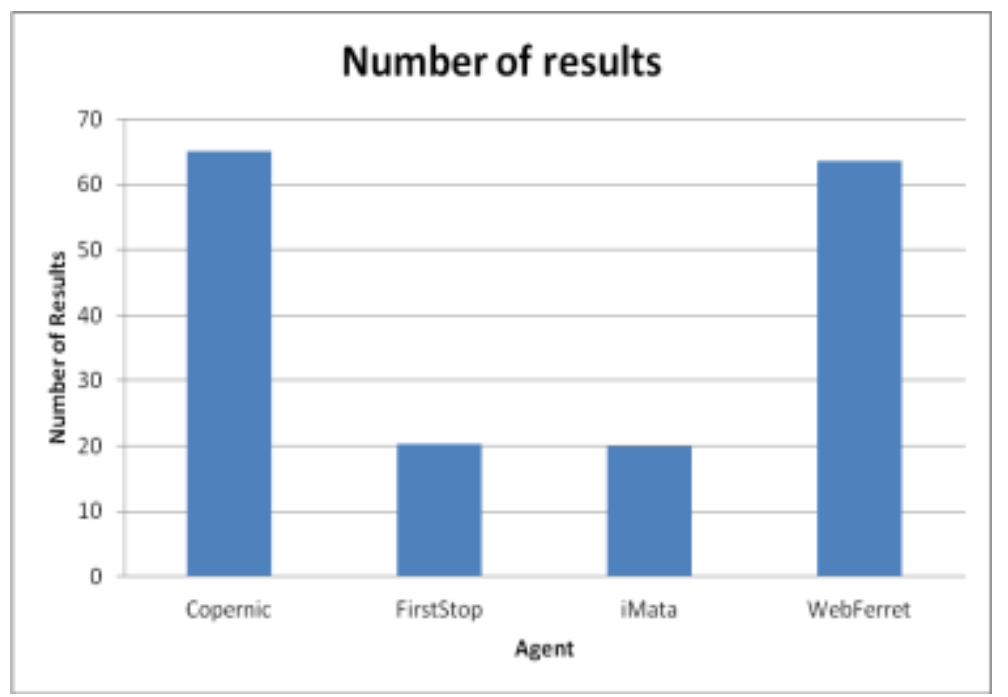

Figure 6. Number of results returned of Copernic, FirstStop, iMeta, and WebFerret

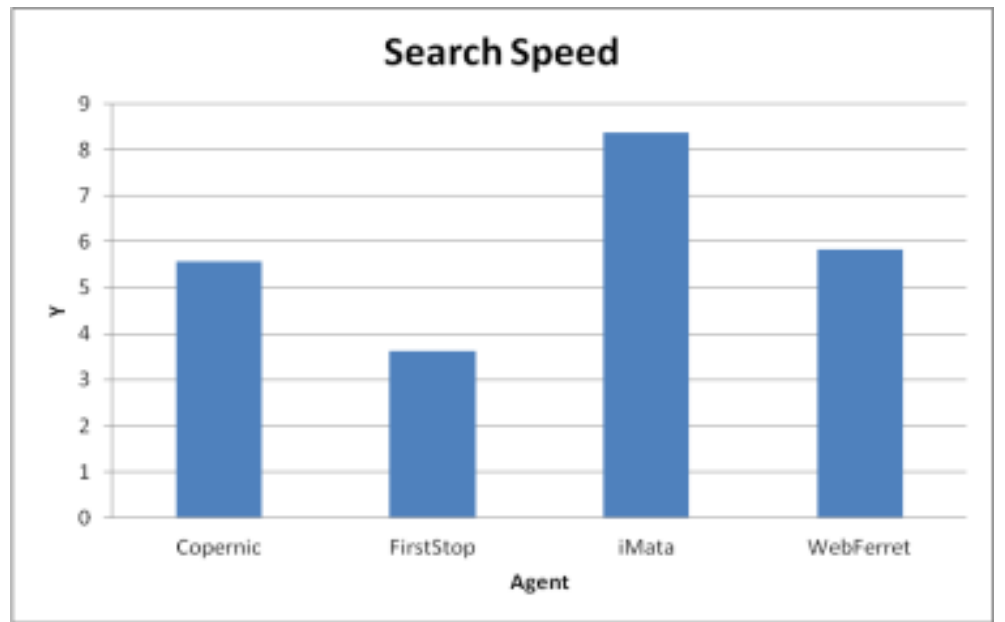

Figure 7. Search Speed Comparison of Copernic, FirstStop Websearch, iMeta, and WebFerret

Table 4 shows the mean search speed with 95\% confidence interval (C.I.) and standard deviation of Copernic, FirstStop Websearch, iMeta, and WebFerret. The 95\% C.I. of each ISA's search speed is shown in the brackets. We found that the standard deviation of search speeds varies significantly from its mean.

Table 4: Mean Search Speed

\begin{tabular}{|c|c|c|}
\hline \multirow{2}{*}{ Search agents } & \multicolumn{2}{|c|}{$\begin{array}{c}\text { Search speed } \\
\text { (results returned/sec/query) }\end{array}$} \\
\cline { 2 - 3 } & Mean (95\% C.I.) & Standard deviation \\
\hline Copernic & $5.56(5.12,6)$ & 2.45 \\
\hline FirstStop & $3.62(3.30,3.94)$ & 1.79 \\
\hline iMeta & $8.38(6.68,10.07)$ & 9.47 \\
\hline WebFerret & $5.83(5.19,6.45)$ & 3.51 \\
\hline
\end{tabular}


To gain additional insight into the impact of complexity of the queries on the performance of ISAs, we carried various experiments with a degree of query complexity. We observed that as the complexity of the queries increases, ISAs tend to lose their search speed. For example, when we used a quotation in the last query (Table 1) to match the exact phrase, all the four ISAs did not perform well.

To analyze the differences in search speeds of the four selected ISAs, the one-way ANOVA (also called F test) was conducted. The idea was to determine the existence of differences among several population means [45]. The results demonstrated that there was a high degree of confidence that at least some of the means differed significantly. The $\mathrm{F}$ value after the ANOVA test was found to be 20.99, which is the ratio of the mean square between agents and the mean square within the agents. The degree of freedom was 4 , which is the variation between the agents. Based on these results, several statistically significant differences in agents mean search speeds were observed.

Figure 8 shows the difference (magnitude) among the search speeds of Copernic, FirstStop Websearch, iMeta, and WebFerret. We observe that iMeta has the highest and FirstStop has the lowest search speed.

\section{Limitations of the Study}

We had downloaded a very limited number of freely available (at no costs) search agents on a local machine for experiments. Due to the budget constraint we could not get hold of any commercial agents to be included in this study.

Because of the complexity in conducting experiments by considering all the variables/parameters involved, the advanced features provided by the ISAs were not used in the investigation, only the default settings were used. Another limitation is that we did not include any human participants in this study. Therefore, performance metrics such as 'accuracy' and 'usability' were not considered both of which are best determined by independent judges especially in relation to the tasks.

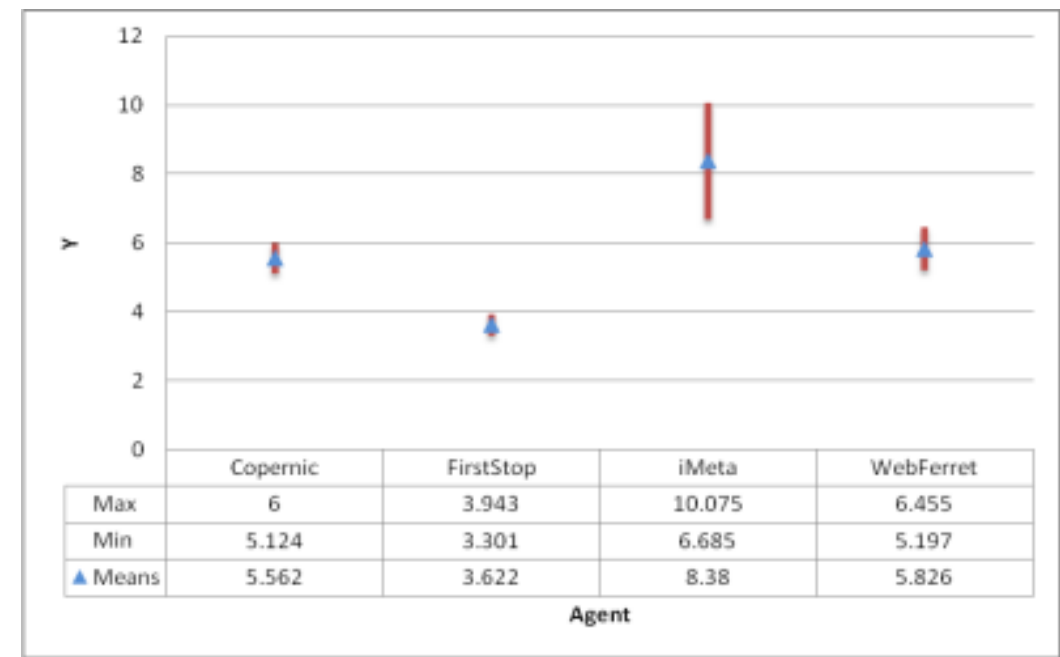

Figure 8. Magnitude of the Difference of Copernic, FirstStop, iMeta, and WebFerret

\section{Conclusion and Future Work}

This paper described an experimental set up for the performance evaluation and comparison of intelligent search agents (ISAs). For an efficient evaluation of ISAs, we introduced a new performance metric called search speed, which is a ratio of the number of results returned per second per query, and RTT. 
By conducting various experiments under a control environment, we gained an insight into the performance evaluation of ISAs. We found that not all ISAs can perform equally well with respect to their information searching capability from the Web. Of the four ISAs evaluated, Copernic is found to be the best which is about $45 \%$ faster than WebFoil. The proposed evaluation method is simple and can be used for evaluating similar search agents. We have tested the system and found to be robust.

To the best of our knowledge this study is one of the first documented attempts for evaluating ISAs. The authors hope that other researchers will continue to explore this field. However, this study can be further extended by using a case study approach in evaluating the performance of similar agents. The impact of query length as well as complexity (e.g., more complex terms) on agent performance is suggested as future research. The performance of ISAs under various data types, such as text, images, voice and video is also planned as an extension of the study reported here.

\section{Acknowledgment}

We thank Bin Li for conducting experiments. This work was supported by National Natural Science Foundation of China under Grant No. 61379027, 51404223, and Dr Li's Postdoctoral Foundation of Jiangsu Grant \# 1104000264. The paper was written when the first author visited China Jiliang University, Hangzhou, China.

\section{References}

[1] N. Boudriga and M. S. Obaidat, "Intelligent agents on the Web: a review", Computing in Science \& Engineering, vol. 6, no. 4, (2004).

[2] R. Dragan, "Software Agents", PC Magazine, vol. 166, no. 9, (1998).

[3] G. Kalakota and A. Winston, "Frontiers of electronic commerce", USA: Addison-Wesley, (1996).

[4] J. Debenham, "An agent-based framework for emergent process management", International Journal of Intelligent Information Technologies, vol. 2, (2006), pp. 30-48.

[5] H. Hamidi and K. Mohammadi, "Modeling fault tolerant and secure mobile agent execution in distributed systems", International Journal of Intelligent Information Technologies, vol. 2, (2006), pp. 21-36.

[6] S. S. Heragu, R. J. Graves, K. Byung-In and A. St Onge, "Intelligent agent based framework for manufacturing systems control”, IEEE Trans on Systems, Man and Cybernetics, Part A, vol. 32, no. 5, (2002).

[7] S. Ramanujam and M. A. M. Capretz, "ADAM: a multi-agent system for autonomous database administration and maintenance", International Journal of Intelligent Information Technologies, vol. 1, no. 3, (2005).

[8] Z. Zi-Ming, M. Bo and Z. Yuan-Yuan, "An intelligent agent-based system in internet commerce”, The International Conference on Machine Learning and Cybernetics. (2005) August 18-21.

[9] J. M. Bradshaw, “An introduction to software agents”, In Software Agents MIT Press, (1997).

[10] S. Franklin and A. Graesser, "Is it an agent, or just a program? A Taxonomy for Autonomous Agents", Third International workshop on agent theories, architectures, and languages, (1996).

[11] S. Machlis, "Agent Technology”, ComputerWorld New Zealand, (1999) May 10.

[12] S. Murugesan, "Intelligent agents on the Internet and Web", IEEE Region 10 International Conference on Global Connectivity in Energy, Computer, Communication and Control, (1998).

[13] H. S. Nwana, "Software agents: an overview", Knowledge Engineering Review, vol. 11, no. 3, (1996).

[14] M. M. Syiam, H. M. Kelash, M. A. Berbar, M. A. Radad and H. M. Faheem, "Getting agents to help with the research", IEEE Potentials, vol. 24, no. 3, (2005).

[15] G. Rimmel, M. Clement and M. Runte, "Intelligent software agents: implications for marketing in ecommerce", The 22nd International IRIS Conference on Enterprise Architectures for Virtual Organizations, Keuruu, Finland, (1999).

[16] Y. Wan, S. Menon and A. Ramaprasad, "A classification of product comparison agents", Communications of the ACM, vol. 50, no. 8, (2007).

[17] T. Saracevic, "Evaluation of Evaluation in Information Retrieval", ACM 18SIGIR, Seattle, USA, (1995), pp. 138-146.

[18] P. Srinivasan, F. Menczer and G. Pantt, "Defining evaluation methodologies for topical crawlers", Paper presented at ACM SIGIR, (2003) Toronto, Canada.

[19] J. Dora, "Evaluating reactive scheduling systems", IEEE/WIC/ACM International Conference on Intelligent Agent Technology, (2004). 
[20] A. Serenko, "Importance of interface agent characteristics from end-user perspective", International Journal of Intelligent Information Technologies, vol. 2, no. 2, (2006).

[21] V. Yoon, B. Broome, R. Singh and T. Guimaraes, "Using agent technology for company knowledge management", Information Resources Management Journal, vol. 18, no. 2, (2005).

[22] W. Pan and I. Hawrysiewycz, "Assisting learners to dynamically adjust learning process through software agents", International Journal of Intelligent Information Technologies, vol. 2, no. 2, (2006).

[23] S. Weiming, "Distributed manufacturing scheduling using intelligent agents", IEEE Intelligent Systems [see also IEEE Intelligent Systems and their Applications], vol. 17, no. 1, (2002).

[24] M. Pantic, R. Zwitserloot and R. J. Grootjans, "Teaching introductory artificial intelligence using a simple agent framework", IEEE Transactions on Education, vol. 48, no. 3, (2005).

[25] W. Shen, S. Y. T. Lang and L. Wang, "iShopFloor: an Internet-enabled agent-based intelligent shop floor", IEEE Transactions on Systems, Man and Cybernetics, vol. 35, no. 3, (2005).

[26] E. Hughes and M. Lewis, "Intelligent agents for radar systems", Electronics Systems and Software, vol. 3 , no. $1,(\mathbf{2 0 0 5})$.

[27] D. Godoy and A. Amandi, "User profiling for Web page filtering", IEEE Internet Computing, vol. 9, no. 4, (2005).

[28] B. J. Jansen, T. Mullen, A. Spink and J. Pedersen, "Automated gathering of web information: an indepth examination of agents interacting with serach engines", ACM Trans on Internet Technology, vol. 6, no. 4, (2006).

[29] J. Salter and N. Antonopoulos, "CinemaScreen recommender agent: combining collaborative and content-based filtering”, IEEE Intelligent Systems, vol. 21, no. 1, (2006).

[30] V. Hajarnavis, "Intelligent agents in manufacturing automation", Paper presented at IEE Seminar on Autonomous Agents in Control, (2005) May 10.

[31] I. Rudowsky, O. Kulyba, M. Kunin, D. Ogarodnikov and T. Raphan, "Managing a relational database with intelligent agents", Paper presented at Int. Conf on IT: Coding and Computing, (2005) April 4-6.

[32] S. Hui-Zhang and Z. Ji-di, "Application of intelligent agent in network payment security", Paper presented at 29th Annual IEEE International conference on Local Computer Networks, (2004) November 16-18.

[33] X. Ji, J. Xu, H. Zhu and Z. Liu, "A realizable intelligent agent model applied in dynamic e-business", Paper presented at the IEEE International Conference on Information Reuse and Integration, (2003) October 27-29.

[34] S. Z. H. Zaidi, S. S. R. Abidi and S. Manickam, "Distributed data mining from heterogeneous healthcare data repositories: towards an intelligent agent-based framework", (2002).

[35] N. Houari and B. H. Far, "Application of intelligent agent technology for knowledge management integration", Paper presented at Third IEEE International Conference on Cognitive Informatics. (2004) August 16-17.

[36] C. Bo, W. Changhong and G. Xiue, "Research on the intelligent agent of distributed fault diagnose system", Paper presented at First International Symposium on Systems and Control in Aerospace and Astronautics, (2006) January 19-21.

[37] B. Kim, S. S. Heragu, R. J. Graves and A. Onge, "Intelligent agent based framework for warehouse control", Paper presented at 37th annual Hawaii Int. Conference on System Sciences, (2004) January 5-8, pp. $1-10$

[38] A. Mawlood-Yunis, A. Nayak, D. Nussbaum and N. Santoro, "Comparing performance of two mobile agent platforms in distributed search", Paper presented at IEEE/WIC/ACM International Conference on Intelligent Agent Technology, (2004).

[39] D. Camacho, R. Aler, C. Castro and J. M. Molina, "Performance evaluation of ZEUS, Jade, and SkeletonAgent frameworks", Paper presented at IEEE International Conference on Systems, Man and Cybernetics, (2002).

[40] D. Qi and R. Sun, "A comparison of team evolution operators", Paper presented at IEEE/WIC/ACM International Conference on Intelligent Agent Technology, (2004).

[41] H. C. Lau and L. Zhang, "A two-level framework for coalition formation via optimization and agent negotiation”, Paper presented at IEEE/WIC/ACM Int. Conference on Intelligent Agent Technology, (2004).

[42] X. Mao, J. Wang and E. Yu, "A framework to specify and evaluate coordination in MAS with logic", Paper presented at the IEEE/WIC/ACM International Conference on Intelligent Agent Technology, (2004).

[43] M. Kaki, "Proportional search interface usability measures", Paper presented at Proportional search interface usability measures, Tampere, Finland, (2004).

[44] B. J. Jansen, "The effects of query complexity on Web searching results", Information Research, vol. 6, no. $1,(\mathbf{2 0 0 0})$.

[45] A. M. Law and W. D. Kelton, "Simulation modelling and analysis", (third ed.), McGraw-Hill, New York, (2000). 


\section{Author}

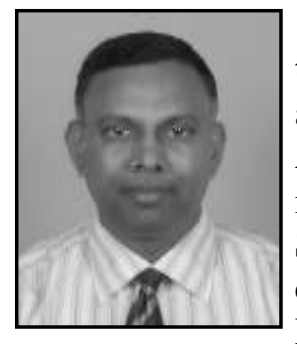

Nurul I Sarkar, (nurul.srakar@aut.ac.nz) holds a PhD from the University of Auckland and is currently associate professor and leader of the Network and Security Research Group at the Auckland University of Technology, Auckland, New Zealand. He is a member of many professional organizations and societies. Dr Sarkar is a regularly invited keynote speaker, chair, and committee member for various national and international fora. He has published over 150 refereed articles and served on the editorial review boards of several prestigious journals. "Improving the Performance of Wireless LANs: A Practical Guide", his second book has been published by Taylor and Francis in January 2014. Dr. Sarkar is a senior member of IEEE. 